\title{
Analyse betriebswirtschaftlicher Kennzahlen zur Unterstützung von Managemententscheidungen
}

\author{
Bertil Haack, Claudia Schmidt
}

\section{Zusammenfassung}

Entscheidungen zu treffen gehört zu den wesentlichen Aufgaben jedes Managers. Die Gefahr von Fehlentscheidungen liegt dabei auf der Hand. Um diese zu vermeiden, ist es von zentraler Bedeutung, eine genaue Analyse der für den Entscheidungsprozess wesentlichen Informationen vorzunehmen. Ziel dieses Papiers ist es, einen Weg aufzuzeigen, wie ein aussagekräftiges Bild der finanzwirtschaftlichen Situation potenzieller Geschäftspartner mithilfe einer fundierten Querschnittsanalyse frei zugänglicher betriebswirtschaftlicher Daten gewonnen werden kann. Dieser Ansatz kann in jeder Entscheidungssituation, in der die Finanzausstattung der betrachteten Unternehmen entscheidungsrelevant ist, genutzt werden.
Abstract

Decision making is one of the most important management tasks. In this context wrong decisions are real risks. In order to avoid them it is necessary to analyse the relevant facts intensively - especially if these decisions affect the selection of a new business partner. Therefore the main goal of this paper is to describe a management analysis using only free accessible data to get a significant impression of the financial situation of potential business partners. This analysis is applicable in each situation where the financial situation of companies is relevant for business decisions.

\section{Einleitung}

Entscheidungen zu treffen gehört zu den wesentlichen Aufgaben jedes Managers. Anlässe dafür gibt es sowohl auf strategischer als auch auf operativer Ebene. So kann neben strategischen Entscheidungen wie die Etablierung einer neuen Niederlassung oder eine Änderung in der Unternehmensstruktur auch die Entscheidung für oder gegen einen Anbieter bestimmter Dienstleistungen Gegenstand eines Entscheidungsprozesses im Unternehmen sein. Während strategische Entscheidungen oftmals Ergebnis eines länger andauernden Prozesses sind, werden operative Entscheidungen häufig kurzfristig und »auf kurzen Wegen « beschlossen. Die Gefahr möglicher Fehlentscheidungen gerade im operativen Bereich ist damit offensichtlich. Auswirkungen können beispielsweise wirtschaftliche Schäden wie langfristig geringere Unternehmensgewinne als erwartet sein. Umso wichtiger ist es, auch auf operativer Ebene eine genaue Analyse der für den Entscheidungsprozess wesentlichen Informationen vorzunehmen. Welche Informationen dabei von zentraler Bedeutung sind, hängt vom Entscheidungsanlass $\mathrm{ab}$.
Der vorliegende Beitrag geht von der realen Situation aus, dass ein Unternehmen $U$ nach einem geeigneten Partner für die Einführung einer SAP-basierten IT-Anwendung sucht. Fünf Anbieter A1 bis A5 mit ähnlicher Geschäfts- und Kundenstruktur befinden sich in der engeren Auswahl begründet durch deren Fachkompetenz und die Erfüllung technischer Mindestanforderungen durch sie. Das gemeinsame Projekt wird etwa 18 Monate dauern und zu erheblichen Veränderungen der Geschäftsprozesse im Unternehmen U führen. Es kann nur dann zu einem Erfolg werden, wenn das Projekt von Anfang bis Ende mit dem gewählten Anbieter durchgeführt werden kann. Dies bedeutet, dass das Risiko einer möglichen Insolvenz des Anbieters während der Projektlaufzeit als Entscheidungskriterium in Betracht zu ziehen ist. Die Auswahl muss deshalb auf einen Anbieter mit angemessener Finanzausstattung fallen. Wie aber kann die wirtschaftliche Lage der Anbieter A1 bis A5 möglichst einfach und stichhaltig geprüft werden?

Ziel der Ausführungen ist es, Managern am gewählten Beispiel »Anbieterauswahl« aufzuzeigen, wie sie in vergleichbaren Entscheidungssituationen vorgehen und mithilfe einer fundierten Querschnittsanalyse frei zugänglicher betriebswirtschaftlicher Daten ein aussa- 
gekräftiges Bild der finanzwirtschaftlichen Situation potenzieller Geschäftspartner gewinnen können.

Dazu werden zunächst die in die Analyse einbezogenen Kennzahlen in ihrer Bedeutung und Aussagekraft dargestellt (Kapitel 2). Anschließend werden die konkreten Werte dieser Kennzahlen für die Anbieter A1 bis A5 ermittelt und für Zwecke der zu treffenden Managemententscheidung miteinander verglichen (Kapitel 3). Die dabei genutzten Echtdaten von A1 bis A5 entstammen dem elektronischen Bundesanzeiger sowie den auf den Webseiten von A1 bis A5 veröffentlichten Geschäftsberichten. Der Beitrag endet mit der Diskussion des Einsatzbereiches der vorgeschlagenen Querschnittsanalyse (Kapitel 4).

\section{Betriebswirtschaftliche Kennzahlen}

Die Analyse der finanzwirtschaftlichen Situation der Anbieter A1 bis A5 basiert auf den folgenden relativen Kennzahlen. Diese lassen sich aus den jeweiligen Bilanzen und Gewinn- und Verlustrechnungen der betrachteten Gesellschaften ermitteln.

- Liquidität

- Eigenkapital- und Fremdkapitalquote (Verschuldungsgrad)

- Anlagendeckungsgrade A und B (Anlagenstruktur)

- Umsatz und Umsatzrentabilität

Dabei ist die Liquidität die für die vorliegende Analyse wichtigste Kennzahl. Es ist Ziel jedes Unternehmens zahlungsfähig zu bleiben (Liquiditätsziel), da andernfalls die Gefahr der Insolvenz und damit das Ende des Unternehmens droht. Genau dieser Umstand soll bei der Wahl eines der potenziellen Anbieter vermieden werden. Die Ermittlung des Verschuldungsgrads wurde als aussagekräftige Kennzahl innerhalb der Querschnittanalyse gewählt, weil damit letztlich eine Aussage über die finanzielle Abhängigkeit des Unternehmens von Anderen getroffen wird und inwieweit sich das Unternehmen selbst tragen kann. Der Umsatz und die Umsatzrentabilität wurden hingegen mit aufgenommen, um aufzuzeigen, dass allein die Umsatzgröße noch kein abschließendes Urteil über die finanzielle Ausstattung eines Unternehmens zulässt und eine Analyse mehrerer Kennzahlen (Querschnittanalyse) von besonderer Bedeutung ist.

Die folgenden Ausführungen belegen, dass die vorgeschlagene Querschnittanalyse bereits weniger Kennzahlen sehr detaillierte Aussagen über die betrachteten
Unternehmen erlaubt. Gleichwohl sei darauf hingewiesen, dass im Bedarfsfall natürlich weitere betriebswirtschaftliche Kennzahlen in die Betrachtungen einbezogen und analog der vorgelegten Diskussion zur Entscheidungsfindung herangezogen werden können.

\section{Liquidität}

Die Liquidität ist Bestandteil der Analyse der Finanzlage eines Unternehmens und besitzt ihre Aussagekraft in den potenziell zur Verfügung stehenden Finanzmitteln eines Unternehmens zur Deckung ihrer kurzfristigen Zahlungsverpflichtungen. Dabei stellt die Liquidität 1 . Grades das Verhältnis zwischen den flüssigen Mitteln und dem kurzfristigen Fremdkapital dar. Die Liquidität 2. Grades bezieht darüber hinaus das übrige monetäre Umlaufvermögen (Umlaufvermögen ohne Vorräte) mit in ihre Berechnung ein. Aus Sicht der Betriebswirtschaft sollte die Liquidität 2. Grades mindestens $100 \%$ betragen (Controller-Portal 2009). Andernfalls ist von einer zu geringen Umschlagshäufigkeit auszugehen. In der Interpretation dieser betriebswirtschaftlichen Kennzahl bedeutet das, dass eine Liquidität 2. Grades kleiner als 100\% für einen Absatz spricht, der die kurzfristigen Verbindlichkeiten des Unternehmens nicht ausreichend zu decken erlaubt. Ist die Liquidität jedoch sehr hoch, so sollten die aus dem Umlaufvermögen freigesetzten liquiden Mittel in renditeträchtige Anlagen investiert werden statt sie zum Ausgleich der kurzfristigen Verbindlichkeiten bereit zu halten. Die Liquiditäten 1. und 2. Grades lassen sich anhand folgender Berechnungsformeln ermitteln (Controller-Portal 2009):

- Liquidität 1. Grades = liquide Mittel: kurzfristige Verbindlichkeiten

Dabei bilden die Guthaben bei der Bank sowie die Kassenbestände die liquiden Mittel. Die kurzfristigen Verbindlichkeiten beinhalten oftmals jene Schulden an Lieferanten, das Finanzamt oder auch die Sozialversicherungsträger. Sie können weiterhin im Rahmen unterjähriger Bankdarlehen entstehen.

- Liquidität 2. Grades = (liquide Mittel + Forderungen): kurzfristige Verbindlichkeiten

Eigenkapital- und Fremdkapitalquote (Verschuldungsgrad)

Beide Kennzahlen sind Bestandteil der Verschuldungsanalyse. Sie werden jeweils als prozentualer Anteil am gesamten Kapital (Summe aus Eigenkapital und Fremdkapital) angegeben (Lexikon der Betriebswirtschaft 2003: 548). Die Fremdkapitalquote wird zudem 
oftmals auch als Verschuldungsgrad bezeichnet, da sie den Schulden des Unternehmens bei seinen Gläubigern wie z. B. Banken, Lieferanten oder Dienstleister entspricht. Es wird deutlich, dass ein Anstieg der Eigenkapitalquote bei gleich bleibendem Gesamtkapital zu einer Reduzierung der Fremdkapitalquote und somit des Verschuldungsgrades führt. Die Aussagekraft beider Kennzahlen liegt insbesondere in der Information, inwieweit das betrachtete Unternehmen finanzwirtschaftlich unabhängig ist. Dennoch ist für eine aussagekräftige Wirtschaftlichkeitsbetrachtung die isolierte Analyse des Eigenfinanzierungsgrades respektive des Verschuldungsgrades nicht zweckmäßig. Mit Hilfe umfangreicherer Kennzahlensysteme kann diese isolierte Betrachtung aufgehoben und können die analysierten Kennzahlen in einen Zusammenhang gebracht werden. So spricht eine hohe Eigenkapitalquote zwar für eine hohe Selbständigkeit hinsichtlich der Finanzierung des Unternehmens. Jedoch ist diese lediglich mit einer entsprechend hohen Wertschöpfung und damit erzielten Rendite auch tatsächlich tragfähig. Die Ermittlung dieser beiden Kennzahlen wird wie folgt vorgenommen:

n Eigenkapitalquote $=($ Eigenkapital $:$ Gesamtkapital $)$ $\mathrm{x} 100$

n Fremdkapitalquote $=($ Fremdkapital $:$ Gesamtkapital $)$ $\mathrm{x} 100$

Anlagendeckungsgrade A und B (Anlagenstruktur) Mithilfe der Analyse der Anlagenstruktur können zum einen Aussagen über die Flexibilität und Entscheidungsfreiheit, zum anderen über die finanzielle Deckung des langfristigen Vermögens gemacht werden. Die finanzielle Flexibilität eines Unternehmens ist demnach umso höher, je größer das Umlaufvermögen ist. Dem gegenüber steht jedoch aufgrund des kurzfristigen Risikos eine geringere Renditechance. Die hier verwendeten Anlagendeckungsgrade A und B geben darüber Auskunft, inwieweit das Anlagevermögen durch langfristiges Kapital (Eigenkapital und langfristiges Fremdkapital) gedeckt ist. Dabei sollte der Richtwert für beide Deckungsgrade $100 \%$ sein. Ein deutlich höherer Deckungsgrad B spricht für eine hohe finanzielle Stabilität des Unternehmens, da neben dem Anlagevermögen auch Teile des Umlaufvermögens durch langfristig zur Verfügung gestelltes Kapital gedeckt ist (ControllerPortal 2009). Die Deckungsgrade A und B sind gegeben durch:

- Anlagedeckungsgrad A = Eigenkapital : Anlagevermögen

- Anlagendeckungsgrad B = (Eigenkapital + Langfristiges Fremdkapital): Anlagevermögen

(Lexikon der Betriebswirtschaft 2003: 548).

\section{Umsatz und Umsatzrentabilität}

Der Umsatz als Ergebnis der Multiplikation der Verkaufsmenge mit dem Verkaufspreis pro Einheit wird häufig als ein zentrales - bzw. sogar einziges - Kriterium bei der wirtschaftlichen Betrachtung eines Unternehmens oder dem Vergleich verschiedener Unternehmen genutzt. Die folgenden Überlegungen zeigen, dass diese absolute Größe allein jedoch kein aussagekräftiges Bild der Lage eines Unternehmens liefert. Wichtig ist wenigstens noch die Betrachtung der Umsatzrentabilität. Diese relative Kennzahl gibt das Verhältnis des Gewinns zum Umsatz an, also den Gewinn pro erzieltem Umsatz.

\section{Analyse frei verfügbarer Unternehmensdaten}

\section{Überblick}

Tabelle 1 gibt einen Überblick über die oben erläuterten Kennzahlen der betrachteten Anbieter A1 bis A5 für das Wirtschaftsjahr 2007. Sie werden anschließend diskutiert.

\begin{tabular}{|c|c|c|c|c|c|}
\hline & A 1 & A 2 & A 3 & A 4 & A 5 \\
\hline Liquidität 1. Grades & $65,92 \%$ & $6,80 \%$ & $42,39 \%$ & $109,45 \%$ & $0,01 \%$ \\
\hline Liquidität 2. Grades & $117,32 \%$ & $232,62 \%$ & $254,95 \%$ & $522,15 \%$ & $350,68 \%$ \\
\hline Eigenkapitalrentabilität & $30,26 \%$ & $21,38 \%$ & $102,91 \%$ & $4,35 \%$ & $85,60 \%$ \\
\hline Gesamtkapitalrentabilität & $12,90 \%$ & $8,45 \%$ & $24,86 \%$ & $2,00 \%$ & $42,44 \%$ \\
\hline EK-Quote & $41,56 \%$ & $29,65 \%$ & $23,80 \%$ & $46,03 \%$ & $49,58 \%$ \\
\hline Verschuldungsgrad & $58,44 \%$ & $70,35 \%$ & $75,97 \%$ & $53,95 \%$ & $50,42 \%$ \\
\hline Anlagendeckungsgrad A & $76,88 \%$ & $213,25 \%$ & $280,93 \%$ & $96,26 \%$ & $218,91 \%$ \\
\hline Anlagendeckungsgrad B & $117,95 \%$ & $433,79 \%$ & $478,55 \%$ & $188,16 \%$ & $344,40 \%$ \\
\hline
\end{tabular}

Tab. 1: Überblick über betriebswirtschaftliche Kennzahlen ausgewählter IT-Anbieter 
Liquidität

Hinsichtlich der Liquidität weist Anbieter 1 mit einer Liquidität 2. Grades in Höhe von 117,32\% das beste Ergebnis auf. Mit seinen zur Verfügung stehenden liquiden Mitteln ist das Unternehmen in der Lage, die kurzfristigen Verbindlichkeiten auszugleichen. Unter Berücksichtigung des zur Verfügung stehenden monetären Umlaufvermögens zeigt es die beste Deckung seiner unterjährigen Schulden auf. Dies spricht für eine schnelle Umschlagsfähigkeit und somit eine zügige Liquidierbarkeit der im Umlauf befindlichen Vermögensteile und lässt einen für das Unternehmen adäquaten Absatz vermuten. Mit 522,15\% und über 300\% liegen Anbieter 4 und Anbieter 5 weit über den gewünschten Ergebnissen von $100 \%$. Ein zu geringer Absatz sowie eine zu hohe Lagerhaltung können hier der Grund sein.

\section{Verschuldungsgrad}

Vergleicht man nun die Kapitalrenditen rückt Anbieter 5 im Ranking weiter nach vorne. Mit einer Eigenkapitalrendite in Höhe von 85,60\% liegt er nur knapp hinter Anbieter 3, der hier eine Rendite in Höhe von 102,91\% erzielte. Jedoch wirkt hier die höhere Eigenkapitalquote von fast $50 \%$ zugunsten des Anbieters 5. Dagegen ist Anbieter 2 hinsichtlich der Mittelherkunft ähnlich wie Anbieter 3 aufgestellt, konnte im Jahr 2007 aber lediglich eine Verzinsung seines eingesetzten Kapitals in Höhe von 21,38\% (Eigenkapitalrendite) respektive $8,45 \%$ (Fremdkapitalrendite) erwirtschaften. Eine aus ökonomischer Sicht optimale Kapitalstruktur weisen mit einem Verhältnis des Eigenkapitals zum Fremdkapital von nahezu 1:1 die Anbieter 1, Anbieter 4 und Anbieter 5 auf. Diese Regel der vertikalen Kapitalstruktur ist in der Betriebswirtschaft die engste Auslegung des Verhältnisses zwischen Eigenkapital und Fremdkapital. Sie ist jedoch nicht als ausschließlich anzusehen. Die Realität zeigt oftmals andere Ergebnisse. So ist eine Eigenkapitalquote von 20 - 25\% wie sie beispielsweise Anbieter 3 aufweist ein durchaus übliches Bild (Ehebrecht/Klein/Krenitz 2006:31).

\section{Anlagenstruktur}

Auch im Hinblick auf die Anlagenstruktur und die Deckungsgrade des Anlagevermögens nehmen die Anbieter 2, Anbieter 3 und Anbieter 5 die vorderen Plätze ein. Mit einem Anlagendeckungsgrad B in Höhe von über $400 \%$ sind die Anbieter 2 und Anbieter 3 auch in wirtschaftlich schlechteren Zeiten finanziell stabil ausgestattet. Hier sind ebenfalls Teile des Umlaufvermögens, welche nicht unmittelbar am Markt in flüssige Mittel umgewandelt werden können, durch das langfristig zur Verfügung gestellte Kapital gedeckt. Abbildung 1 zeigt die Unterschiede der Anlagendeckung und damit der finanziellen Stabilität auf.

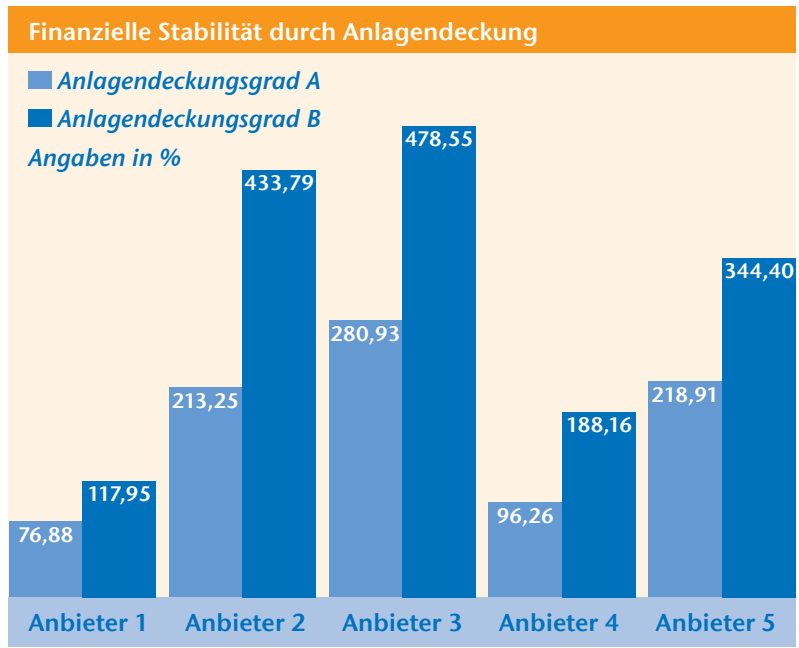

Abb. 1: Finanzielle Stabilität durch Anlagendeckung

\section{Umsatz und Umsatzrentabilität}

Die hier vorgelegte Querschnittanalyse wird durch die Betrachtung der »klassischen« Kennzahlen Jahresumsatzerlös und Jahresüberschuss abgerundet. Zur Ermittlung des Jahresüberschusses kommen das Umsatzkosten- und das Gesamtkostenverfahren in Betracht. Beide Verfahren unterscheiden sich zwar in ihren Ermittlungsschemata, liefern jedoch im Ergebnis den gleichen Wert. Dabei kann der Überschuss der Erträge über den Aufwendungen einen Verlust oder einen Gewinn darstellen. Tabelle 2 zeigt die Umsatz- und Kostenstruktur der betrachteten Unternehmen.

\begin{tabular}{|l|r|r|r|r|r|}
\hline & A 1 & A 2 & A 3 & A 4 & A 5 \\
\hline Jahresumsatz (in TEUR) & 157.311 & 99.290 & 7.602 & 18.432 & 9.086 \\
\hline Kosten (in TEUR) & 147.426 & 98.239 & 6.883 & 18.153 & 8.230 \\
\hline EBIT (in TEUR) & 16.720 & 1.051 & 719 & 279 & 856 \\
\hline Umsatzrentabiliät (in \%) & 10,63 & 1,06 & 9,46 & 1,52 & 9,42 \\
\hline
\end{tabular}

Tab. 2: Umsatz- und Kostenstruktur der potenziellen Anbieter 
Es wird deutlich, dass die Umsatzhöhe allein noch keine aussagekräftige Interpretation des ökonomischen Handelns eines einzelnen Anbieters zulässt. Anbieter 1 und Anbieter 2 erzielten im Jahr 2007 den höchsten Umsatz, wohin gegen Anbieter 3 mit einem Umsatz von 7.602.249 Euro das Schlusslicht bildet. Dennoch war es Letzterem möglich eine Umsatzrendite in Höhe von 9,36\% zu erwirtschaften. Dagegen erwirtschaftete Anbieter 2 lediglich eine Umsatzrendite in Höhe von $1,06 \%$. Damit weist Unternehmen 3 trotz des vergleichsweise geringeren Umsatzes eine ausgewogene Kostenstruktur aus.

\section{Bewertung der Ergebnisse}

Zusammen mit den Jahresumsatzerlösen und -überschüssen erlauben die oben dargestellten betriebswirtschaftlichen Kennzahlen eine Bewertung der wirtschaftlichen Lage eines Unternehmens. Die folgende Tabelle gibt eine Auswertung der Kennzahlen in Form einer Rangliste. Dabei wird pro Kennzahl eine Platzierung (1 bis 5) der Anbieter vorgenommen.

\begin{tabular}{|l|l|l|l|l|l|}
\hline & A 1 & A 2 & A 3 & A 4 & A 5 \\
\hline Liquidität & 1 & 2 & 3 & 5 & 4 \\
\hline Verschuldungsgrad & 3 & 4 & 2 & 5 & 1 \\
\hline Anlagendeckungsgrad & 5 & 2 & 1 & 4 & 3 \\
\hline Umsatz & 1 & 2 & 5 & 3 & 4 \\
\hline Umsatzrentabilität & 1 & 5 & 2 & 4 & 3 \\
\hline
\end{tabular}

Tab. 3: Entscheidungsmatrix und Auswertung der analysierten Kennzahlen

Tabelle 3 zeigt, dass die Betrachtung einer einzelnen Kennzahl eine Fehlentscheidung des Managements herbeiführen kann. Würde man beispielsweise lediglich den Umsatz der potenziellen Anbieter berücksichtigen, würde Anbieter 3 ausgeschlossen werden. Unter Berücksichtigung der Umsatzrentabilität und des Verschuldungsgrades kann Anbieter 3 jedoch durchaus als möglicher Favorit betrachtet werden. In diesem Licht erscheint eine Entscheidung des Unternehmens U für diesen Anbieter durchaus sehr sinnvoll . Es wird deutlich, dass die Analyse mehrerer Kennzahlen von entscheidender Bedeutung ist, um ein aussagekräftiges Bild der potenziellen Anbieter bzw. Geschäftspartner zu erhalten.

\section{Einsetzbarkeit und Fazit}

Das vorliegende Papier stellt eine Querschnittanalyse vor, mit der quantitative Informationen über die ökonomische Lage eines oder mehrerer Unternehmen gewonnen werden können.

Dieser Ansatz zeichnet sich einerseits durch seine Einfachheit und den geringen erforderlichen Aufwand aus. Andererseits basiert er auf frei verfügbaren Informationen über die betrachteten Unternehmen und liefert ein ausgesprochen differenziertes Bild von ihnen.

Der Vergleich der gewonnenen Kennzahlen mehrerer Unternehmen erlaubt, diese Gesellschaften hinsichtlich ihrer wirtschaftlichen Stabilität einzuordnen und so eine anstehende Entscheidung für oder gegen eines dieser Unternehmen als Geschäftspartner zu unterstützen. Würde man bei der Auswahl eines IT-Anbieters und der für diese Zwecke durchgeführten Analyse seiner finanziellen Stabilität beziehungsweise Leistungsoder gar Überlebensfähigkeit lediglich auf die betriebswirtschaftlichen Kennzahlen »Jahresumsatzerlös « und »Jahresüberschuss« Wert legen, käme ein wenig differenziertes und möglicherweise verfälschtes Bild zustande. Anhand der hier untersuchten IT-Dienstleister A1 bis A5 wird deutlich, dass tatsächlich auch die Anbieter, deren Jahresergebnisse relativ zum Wettbewerb geringer ausfallen, an der Spitze des Rankings stehen oder zumindest mit den vermeintlich Großen der Branche mithalten können.

Insgesamt wird Führungskräften damit ein Entscheidungskriterium und dessen richtige Beurteilung an die Hand gegeben, welches sie - wie etwa im vorliegenden Beispiel - zur Einschätzung von IT-Dienstleistern, aber etwa auch von Lieferanten, möglichen Kooperationspartnern o. ä. in jedweder Branche nutzen und deren Ergebnisse sie zur Untermauerung ihrer Entscheidungen für oder gegen eine Zusammenarbeit mit diesen Unternehmen heranziehen können. 


\section{Literaturverzeichnis}

Controller-Portal 2009

http://www.controllingportal.de/Fachinfo/Grundlagen/Kennzahlen/ deckungsgrad1.html, (Zugriff 13.04.2009).

http://www.controllingportal.de/Fachinfo/Grundlagen/Kennzahlen/ liqui2.html, (Zugriff 19.04.2009).

http://www.controllingportal.de/Fachinfo/Grundlagen/Kennzahlen/ Personalintensitaet.html, (Zugriff 19.04.2009)

Ehebrecht, H.-P., Klein, V., Krenitz, M. (2006): Finanzierung und Investition, Stam Verlag, Köln, 2006

Haack, B., Neumann, I. (2005): IT-Landschaft im Wandel - Veränderungsprozesse in der Wohnungswirtschaft, PROMOS Press, Berlin, 2005

Schneck, O. (Hrsg.) (2003): Lexikon der Betriebswirtschaft, Beck Verlag, München, 2003.
Autoren

Prof. Dr. Bertil Haack

Dekan

Fachbereich Wirtschaft, Verwaltung und Recht

Technische Hochschule Wildau [FH]

bertil.haack@th-wildau.de

\section{Claudia Schmidt, M.A.}

Europäisches Management

Fachbereich Wirtschaft, Verwaltung und Recht

Technische Hochschule Wildau [FH]

schmidt-claudia80@arcor.de 